\title{
Dynamic spectrum access using markov chain technique for regional area network
}

\author{
Jayant P. Pawar ${ }^{1}$, Prashant V.Ingole ${ }^{2}$ \\ ${ }^{1}$ Jayant P. Pawar, Research Scholar, Sant Gadge Baba Amaravati University, India \\ ${ }^{2}$ Dr. Prashant V. Ingole, HOD, Dept. of IT, PRMIT\&R, India
}

\begin{tabular}{l} 
Article Info \\
\hline Article history: \\
Received Nov 31, 2019 \\
Revised Feb 1, 2020 \\
Accepted Feb 15, 2020 \\
\hline
\end{tabular}

Keywords:

Cognitive radio network

Dynamic spectrum access

Markov chain

Regional area network

Utilization factor

\begin{abstract}
Cognitive Radio Network is the effective solution to the spectrum scarcity. Dynamic spectrum access is a paradigm used to access the spectrum dynamically. A Markov Chain is a stochastic model describing a sequence of possible events in which probability of each event depends only on the state attained in the previous event. We model the dynamics of cognitive user with 2-D Markov chain. The resource distribution probability (RDP) verses addition/ elimination rate of the channels in the network is also plotted. The RDP verses utilization factor of the queue, which is the secondary user in the network, is also plotted. This plot helps to maintain the total arrival and departure rate based on the RDP. The base station of the network will use this relation to maintain the proper RDP for the devices. The dynamics of each cognitive user and its correlation with Markov Chain is an interesting approach. Here we considered the DSA at Base Station as a Markov chain and analyzed it. This analysis helps us to determine the behavior of the cognitive radio and also helps to find the fault in the cognitive devices.
\end{abstract}

Copyright $(0) 2020$ Institute of Advanced Engineering and Science. All rights reserved.

\section{Corresponding Author:}

Jayant P. Pawar,

Department of Applied Electronics, SGB, Amaravati University, India. Email: jayant @rgcetpdy.ac.in

\section{INTRODUCTION}

Wireless technology is developing rapidly, and offering many societal and individual benefits. The output of this technology is more penetrative because of many consumer devices such as cell phone, laptops and PDAs are commonly used. The explosion of wireless applications creates an ever increasing demand for more radio spectrum. The spectrum is fixed and only the technology can modify to meet these demands. Broadband is unlocking vast new possibilities for existing ones and enabling entire new industries. There is a paradigm shift in this age in: how we manage energy, deliver health care services, educate children, ensure public safety, engage government etc. Cognitive radios [1] offer the promise of being innovative which will enable the future wireless works. Cognitive radios are fully programmable wireless devices that can sense their environment and dynamically adapt their channel access method, transmission waveform, spectrum use and networking protocols. Dynamic spectrum access (DSA) [2-5] is a new spectrum sharing paradigm that utilizes the spectrum holes and hence increases spectrum utilization as well as alleviates the spectrum scarcity problem.

The IEEE standard for cognitive wireless regional area networks (WRAN) IEEE 802.22 [6-9] is the first cognitive standard release by IEEE. The most prominent target application of 802.22 is wireless broadband access in remote and rural areas. The study and analysis of WRAN using IEEE 802.22 standard is one of the objective of this paper. DSA has three states, sensing, channel sharing and mobility. The problem is how to analyze this process so that the cognitive devices' functionality can be assessed. We applied the Markov Chain [10-13] technique to study the behavior of DSA in cognitive radio. This analysis helps us to 
detect the errors in the cognitive device. Single channel [14] and multichannel heterogeneous cognitive networks [15-18] analysis is important for future network development.

The rest of the paper is organized as follows. In section 2, IEEE 802.22 is explained with data using software ${ }^{\circledR}$ NETSIM version 8.2. In section 3 the modeling of WRAN at the MAC layer is elaborated using Jackson network [19] and theory of queue [20-25]. In section 4 DSA analysis of CR at Base Station of WRAN is explain using Markov Chain. In section 5 the conclusion is given.

\section{IEEE 802.22WIRELESS REGIONAL AREANETWORK}

The scenario used is shown in Figure 1. The following data is calculated by setting the source Id (6) and destination Id (9). The cognitive users (CPEs) are connected to their respective base station. As the operation time is constant and operational interval is increased the opportunity for CR user to access the channels is more. Here the operational interval is varied and its effect on the payload transmitted for different number of incumbents is shown in the Figure 2 and Table 1.This graph is for Base Station 1. It is clear from the graph that payload increases with operational interval. It is also true that payload increases with number of incumbents.

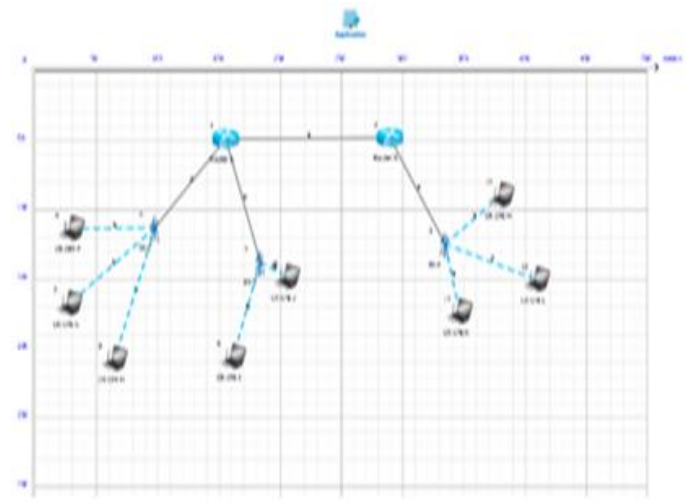

Figure 1. System scenario



Figure 2. Payload verses operational interval for different number of incumbents

Table 1. Cognitive radio channel metrics for different values of incumbents

\begin{tabular}{cc}
\hline Operational interval & Payload transmitted for no of incumbent 1 \\
\hline 3 & 1368304 \\
7 & 2334344 \\
10 & 4213004 \\
operational interval & payload transmitted for no of incumbent 2 \\
3 & 1512534 \\
7 & 3032700 \\
10 & 4271314 \\
operational interval & payload transmitted for no of incumbent 3 \\
3 & 1951294 \\
7 & 3514464 \\
10 & 4361684 \\
\hline
\end{tabular}

\section{MODELING OF WRAN}

The total number of sub channels in the network is denoted by $n$. The input process to the CPE is a stream of data with average rate $\lambda$. The queuing policy is first-in first out (FIFO).

In this work following notations are used:

a) Accumulative inaccuracy factor for each CPE:1- $\mathrm{P}_{\mathrm{s}, \mathrm{i}}$, for $\mathrm{i}=1,2, \ldots \mathrm{m}$

b) Probability, $\mathrm{P}_{\mathrm{s}, \mathrm{i}}$. This is the probability of data packets from CPE already embarked on a server during upstream subframe (US)interval gets transferred to BS.

c) $\mathrm{P}_{\mathrm{s}}$ : probability of data packets from $\mathrm{BS}$ already embarked on a subchannel during downstream subframe (DS) interval gets transfer to CPE.

Here the WRAN as a Jackson network with an M/M/m queue is considered. 
Now, if only one CPE exists in the WRAN cell, then one can model it with for 2-D CTMC \{(E (t), $\mathrm{x}$ (t)): $\mathrm{t}>\mathrm{o}$ \} as shown in Figure 3 [7].

This CTMC models the dynamics of single CPE. It evolves in two directions. The horizontal transitions with rates: $\lambda \mathrm{i}$ and $\mu \mathrm{i}$ stand for the total arrival and total departure of packets in the queue respectively. Vertical transitions with rates $\Pi \mathrm{i}$ and $\xi \mathrm{i}$ represent the addition and elimination of a subchannel that has been announces available by the BS during the US/DS Maps. The state $\left(\prod_{\mathrm{i}}, \mathrm{j}\right)$ of this Markov Chain are samples of the state space where Пirepresents that total numbers of allocated subchannels and $\mathrm{j}$ represents the number of data packets stacked in its queue. The stability condition for the CTMC shown in Figure 3 is,

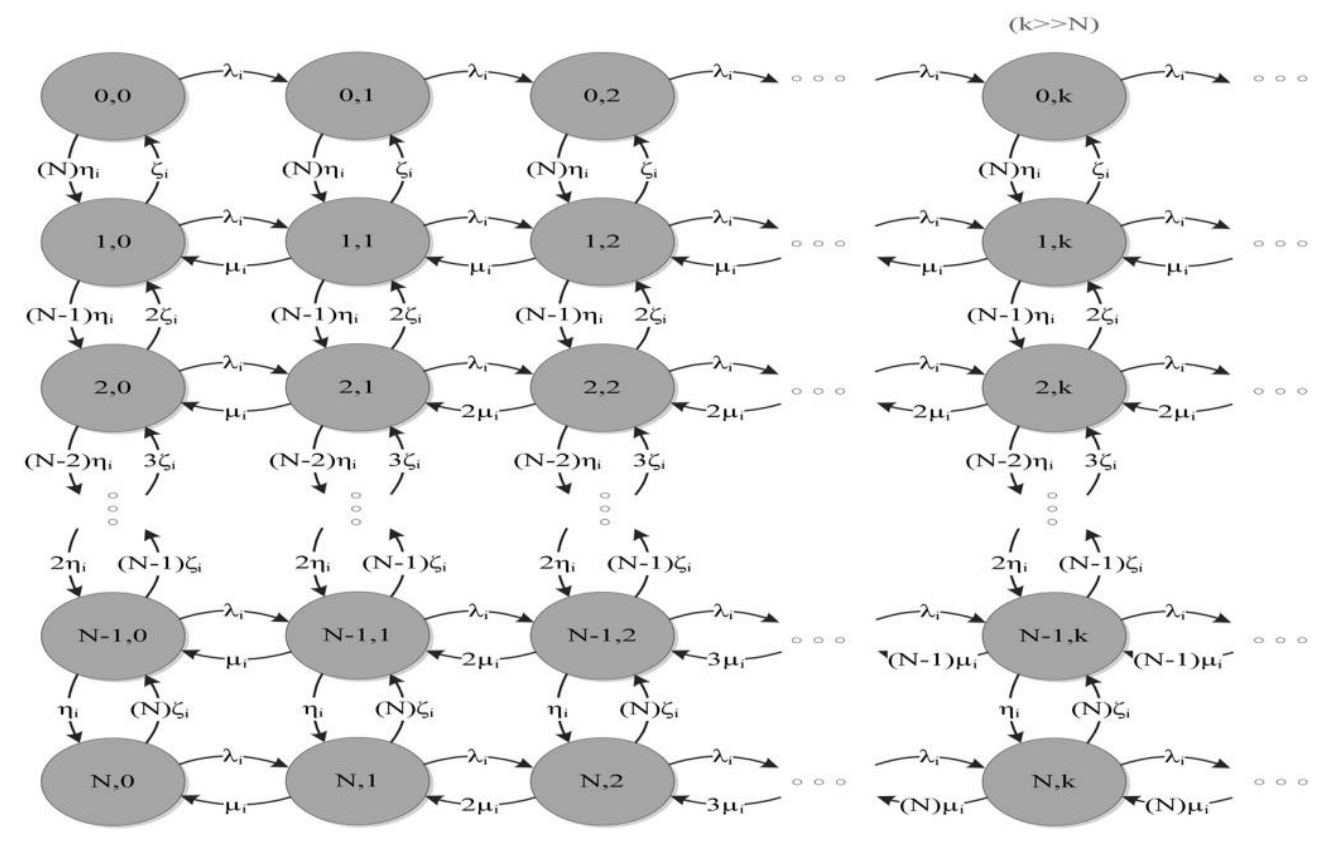

Figure 3. 2-D CTMC representation for the ithtagged CPE [7]

$$
\frac{\lambda_{i}}{\mu_{i}}<N\left(\frac{\eta_{i}}{\xi_{i}+\eta_{i}}\right)
$$

Where $\mathrm{N}=\left(1-\mathrm{P}_{\text {Busy }}\right) \mathrm{n}[7]$.

$\mathrm{N}$ is the average number of available subchannels that can be assigned to a tagged CPE. Now the total number of $\mathrm{N}$ subchannels should be appropriately distributed among $\mathrm{m}$ CPEs. Assuming that $\mathrm{N}$ subchannels are to be allocated to $\mathrm{m}$ associated CPE, with distributing probabilities $\mathrm{q}_{1}, \mathrm{q}_{2}, \ldots, \mathrm{q}_{\mathrm{m}}$ where $\sum \mathrm{q}_{\mathrm{i}}=1$. The probability that the partitioning $\mathrm{n}_{1}, \mathrm{n}_{2} \ldots \mathrm{n}_{\mathrm{m}}$ is chosen among al possibilities would be equal to

$$
P\left(X_{1}=n_{1}, \ldots, X_{m}=n_{m}\right)=\frac{n !}{n_{1} ! . n_{2} ! \ldots n_{m} !} \cdot q_{1}^{n_{1}} \cdot q_{2}^{n_{2}} \ldots q_{m}^{n_{m}}
$$

$$
\sum \mathrm{nm}=\mathrm{N}
$$

This is a multinomial distribution. It is $i^{\text {th }}$ marginal distribution i.e. the number of servers allocated to the CPE would be binomially distributed as $\mathrm{B}\left(\mathrm{n}_{\mathrm{i}}, \mathrm{q}_{\mathrm{i}},\right) \mathrm{i}=1, . ., \mathrm{m}$,

Also each CPE in the network can be modeled with a separate 2-D CTMC similar to that of Figure 3. So $(\mathrm{m}+1)$ 2-D CTMC (m CPEs and one BS) will absolutely model the network.

\subsection{Choice of $q_{i}$}

The vertical transitions in Figure 3 might change at the end of every frame (in IEEE 802.22, it is 10 $\mathrm{ms})$, the suitable choice for $\eta_{\mathrm{j}}$ and $\xi_{\mathrm{i}}$ such that $\eta_{\mathrm{i}} /\left(\eta_{\mathrm{i}}+\xi_{\mathrm{i})}=\mathrm{q}_{\mathrm{i}}\right.$ gives the following results [7]. 


$$
\eta_{i}=\frac{q_{i}}{T_{F}} \text { and } \xi_{i}=\frac{1-q_{i}}{T_{F}}, i=1,2, . ., m
$$

Where $\mathrm{T}_{\mathrm{F}}$ is a frame length.

The choice for resources distribution probabilities (subcarrier distribution probabilities) among CPEs, i.e. $q_{i}$ and $P_{s, i}$ are an important consideration. Several approaches are available in the literature [7].

1) SER-based linear weighting:

In this case the highest amount of allocation goes to the CPE with the most successful transmission history. Hence.

$$
q_{i}=\frac{1-P_{s, i}}{\sum_{j=1}^{m}\left(1-P_{s, i}\right)}
$$

Here, $\mathrm{P}_{\mathrm{s}, \mathrm{i}}$ links with symbol error rate (SER) at its corresponding CPE.

2) Detection-based linear weighting.

Considering IEEE 802.22, BS receives the local sensing results from CPE, it has a good estimate on the detection probabilities of all the CPEs. Here BS will allocate more subchannels to the CPE that has shown higher detection probability $\mathrm{P}_{\mathrm{d}, \mathrm{i}}$. In this case $\mathrm{q}_{\mathrm{i}}$ can be set as,

$$
q_{i}=\frac{P_{d, i}}{\sum_{j=1}^{m} P_{d, j}}
$$

3) Outage -based linear weighting.

In this case the highest amount of allocation goes to the CPE that is closer to the BS hence produces the less interference to the primary receiver.

By defining, the outage probability is given by $\mathrm{P}_{\text {out }}, \mathrm{i}=\mathrm{P}\left(\mathrm{SNR}_{\mathrm{i}}<\left(\mathrm{SNR}_{\min }\right)\right.$. Therefore.

$$
q_{i}=\frac{1-P_{o u t}}{\sum_{j=1}^{m}\left(1-P_{o u t, j}\right)}
$$

\subsection{Cell stability condition}

The general condition for non-saturated operation is described in [7]. The CPEs and BS should have a limited utilization factor $\rho=\lambda_{\mathrm{i}} / \mu_{\mathrm{i}}$ all the time, such that,

$$
\begin{gathered}
\rho_{i}=\frac{\lambda_{i}}{\mu_{i}}<N . q_{i}, i=1,2, \ldots, m . \\
\rho_{B S}^{U S}=\frac{A_{B S}^{U S}=\sum_{i=1}^{m} \lambda_{i}}{\mu_{B S}}<1
\end{gathered}
$$

These conditions, if met simultaneously should guarantee the seamless functioning of the network,

$$
\rho_{B S}^{D S}=\frac{A_{B S}^{D S}=A}{\mu_{B S}}<N
$$


Where, $A_{B S}^{D S}$ is a data rate from backbone network to BS and $A_{B S}^{U S}$ is data rate of BS to backbone network Summing the above equations,

$$
\rho_{B S}^{D S}+\rho_{B S}^{U S}+\sum_{i=1}^{m} \rho_{i}<2 N+1
$$

This equation indicated the stable operation. It means for stable operation, the sum of utilization factors should be smaller than twice the number of subchannels. Figure 5 shows the stability condition for the CTMC process. Here the utilization factors or queue stress increases along with distribution probability $\left(\mathrm{q}_{\mathrm{i}}\right)$. Figure 4 shows the graph for the different values of average number of available subchannels that can be assigned to a tagged CPE verses distribution probability of that CPE. Figure 5 shows the addition / elimination rate verses distribution probability. We can observe that if distribution probability is 0.5 then addition and elimination rate become same, it means that queue length is not varying otherwise it is varying.

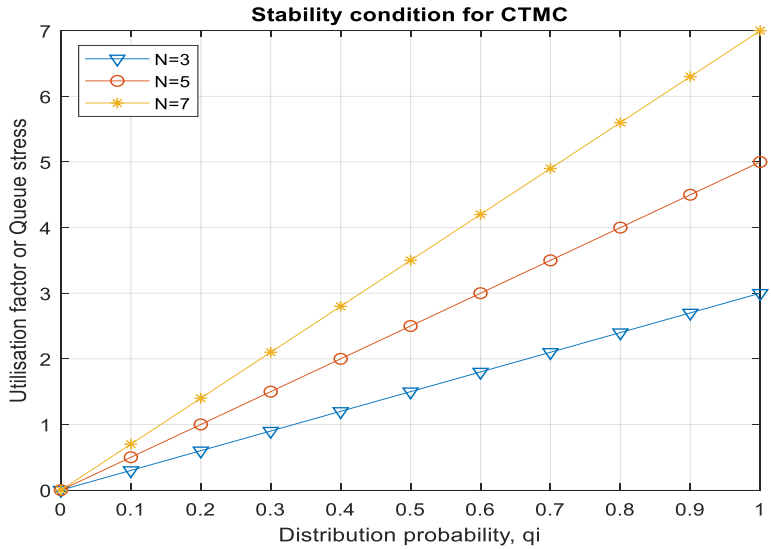

Figure 4. Stability condition for CTMC

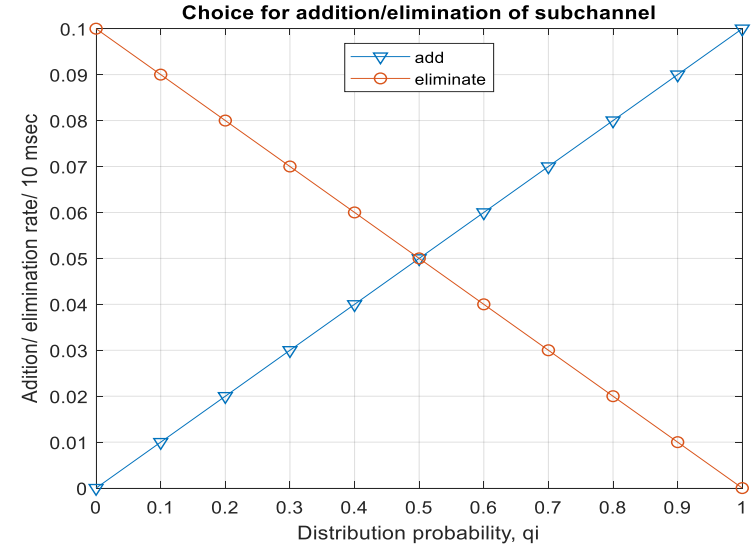

Figure 5. Choice for addition/elimination of channel

\section{MARKOV CHAIN ANALYSIS OF DSA OF COGNITIVE RADIO}

The precise definition of MC varies, for example it is common to define Markov chain as a Markov process in either discrete or continuous time with a countable state space. It is also common to define it as having discrete time in either countable or continuous state space. Here we consider the Markov chain states as a state of the cognitive radio of Base Station of the WRAN. State 1 indicates the spectrum sensing and decision making state of the radio. State 2 indicates the spectrum sharing state. State 3 indicates the mobility state of the DSA. It is shown in Figure 6. We create the two MCs using transition probability matrix. Figure 7 and Figure 8 shows the eigenvalues of Markov chain 1 (MC1) and Markov chain 2 (MC2) on complex planes. Figure 9, shows the comparison of stationary distribution of MC1 and MC2.

An eigenvalue plot indicates whether the Markov chain is periodic, and the plot reveals the period of the chain. All eigenvalues at roots of unity indicate the periodicity. The spectral gap is the area between the circumference of the unit circle and the circumference of the circle with a radius of the second largest eigenvalue magnitude (SLEM). The size of the spectral gap determines the mixing rate of the Markov chain. The mixing time of a Markov chain is the time until the Markov chain is "close" to its steady state distribution. In general, the spectrum determines structural properties of the chain.

A stationary distribution of a MC is a probability distribution that remains unchanged in a MC as it progresses. Stationary distribution of MC gives us the important confirmations like transient state and recurrent stat. Transient state indicates the state never reached and recurrent states form the groups and do not communicate with each other. The stationary distribution represents the limiting time-independent distribution of the state for Markov process as the number of steps on transition increases. 


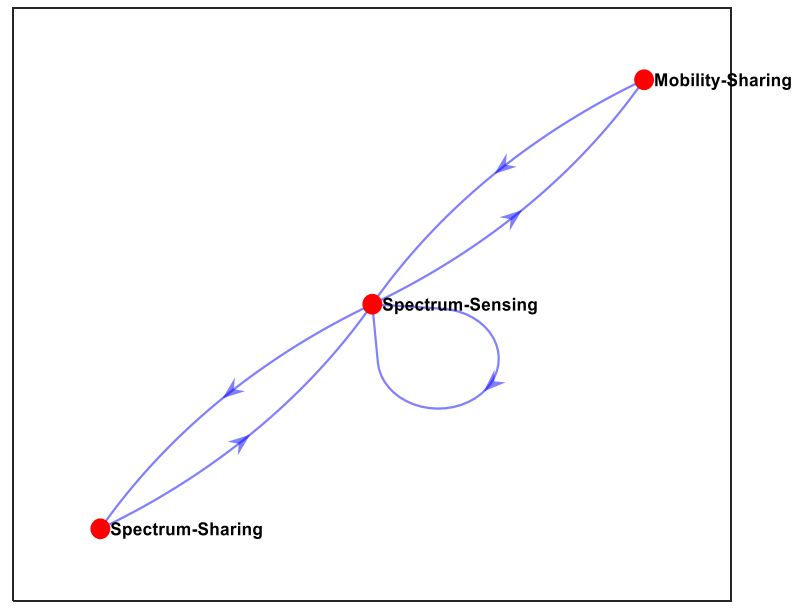

Figure 6. Markov Chain of DSA

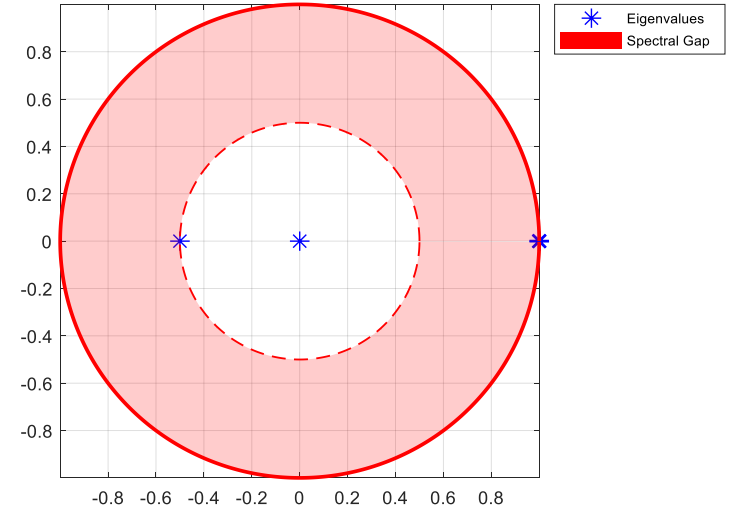

Figure 7. Eigenvalues of MC1on complexplanes

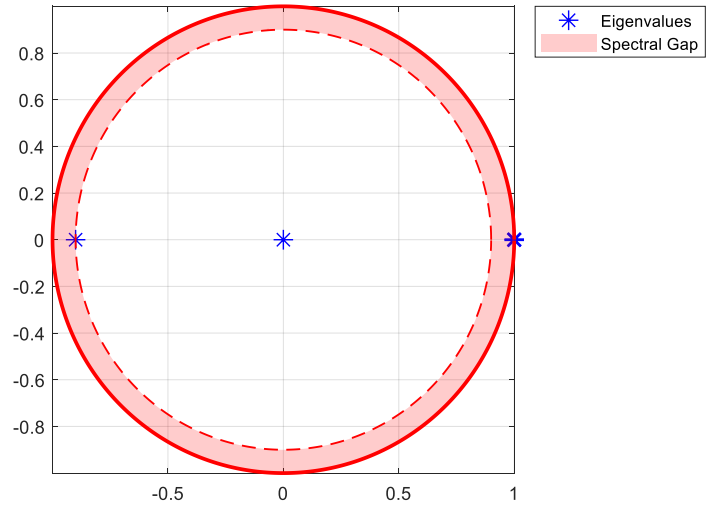

Figure 8. Eigenvalues of MC2 on complex planes

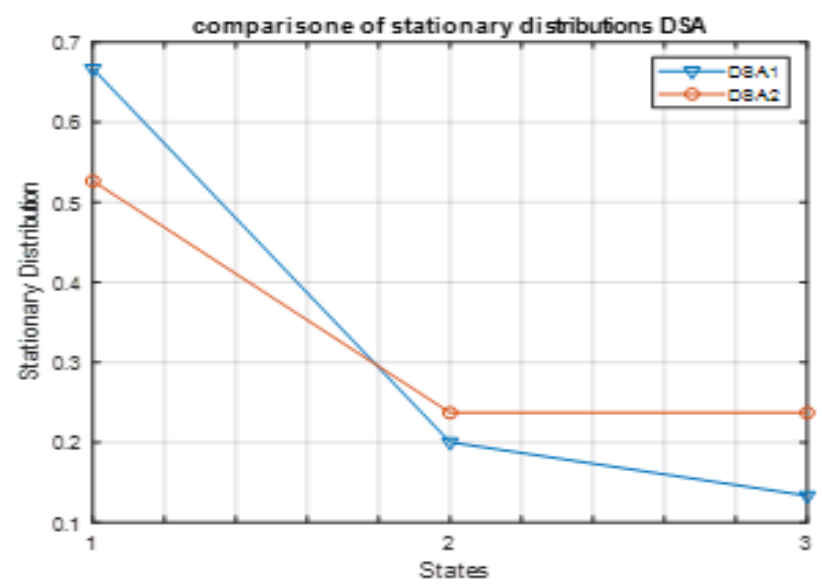

Figure 9. Stationary distributions of two MCs

\section{CONCLUSION}

In this paper we have proved the relation between number of incumbents and payload transmitted in the IEEE 802.22 WRAN network. Also if we increase the operational intervals, payload transmitted gets increase.

Dynamic spectrum access using markov chain technique for regional area network (Jayant P. Pawar) 
In the modeling of the network, we have plotted the maximum utilization factor for stable operation of the network. Queue length of the device is generally fixed, so the selection of the best tradeoff between total number of sub channels and distribution probability increases the network efficiencies. Also plotted addition/elimination rate verses distribution probability possible in the network. When the distribution probability increases, sub-channel addition rate also increases proportionally. Reverse is also true, when the distribution probability decreases, subchannel elimination rate also get increasing. The frame length (US/DS Map) in time domain decides the slope of the graph. So distribution probability plays an important role in the mechanism of DSA in the device.

In this work the dynamics of each cognitive user and its correlation with Markov Chain is studied. Eigen values are instrumental to understand the system. It tells us about the systems that evolve step by step Both the MCs have one Eigen value at unity. Because one eigenvalues are on the unit circle, the chains have a period of 1. Also it shows the spectral gap associated with these eigenvalues. This spectrum determines structural properties of MC such as periodicity. Also from spectral gap we can conclude the mixing rate of $\mathrm{MC} 1$ is greater than MC2 i.e. MC2 is slower than MC1.From a stationary distribution of a MC we conclude that there is no transient state or recurrent state in both the MCs. Transient state indicate the state never reached and recurrent states form the groups and do not communicate with each other, this information helps us to find the fault in the cognitive devices in the network.

\section{ACKNOWLEDGMENT}

The authors would like to acknowledge the support provided by department of applied electronics, Sant Gadge Baba Amravati University, Maharashtra, India.

\section{REFERENCES}

[1] Mitola, J., III; Maguire, G.Q. Jr., "Cognitive radio: making software radios more personal," IEEE Personal Communications, Vol. 6, no. 4 pp. 13-18, Aug 1999.

[2] Qing Zhao Sadler, B.M., “A Survey of Dynamic Spectrum Access,” IEEE Signal Processing Magazine Vol. 24, No. 3, pp. 79-89, May, 2007.

[3] M. M. Buddhikot, "Understanding Dynamic Spectrum Access: Models, Taxonomy and Challenges," Proc. IEEE DySPAN, Apr. 2007.

[4] C.-J. Kim et al., "Dynamic Spectrum Access/ Cognitive Radio Activities in Korea," Proc. IEEE DySPAN, Apr. 2010.

[5] F. C. Commission, "Spectrum policy task force: findings and recommendations," in 2002 International Symposium on Advanced Radio Technologies, ser. 02-13, Nov. 2002.

[6] IEEE 802.22, "Part 22: cognitive wireless RAN medium access control (MAC)and physical layer (PHY) specifications: policies and procedures for operation in the TV bands," Jun. 2011.

[7] N. Tadayon and S. Aissa, "Modeling and Analysis of Cognitive Radio Based IEEE 802.22 Wireless Regional Area Networks," IEEE Transactions on Wireless Communications, Vol 12, NO 9, pp 4363-4375, September 2013.

[8] A. Mody, "Spectrum sensing of the DTV in the vicinity of the pilot using higher order statistics," IEEE 802.22 contribution Doc. IEEE 802.2207/0370r3, Aug. 2007.

[9] J. Zhao, H. Zheng, and G. Yang, "Distributed coordination in dynamic spectrum allocation networks," in Proc. 2005 IEEE DySPAN, pp. 259-268, 2005.

[10] Markov, A. A., “ Theory of Algorithms," [Translated by Jacques J. Schorr-Kon and PST staff] Imprint Moscow, Academy of Sciences of the USSR, 1954.

[11] K.B. Athreya and P. Ney, "A new approach to the limit theory of recurrent Markov chains", Trans. Amer. Math. Soc. Vol. 245, 493-501, 1978.

[12] J.A. Fill, "Eigenvalue bounds on convergence to stationarity for nonreversible Markov chains, with an application to the exclusion process", Ann. Appl. Prob. Vol. 1, 64-87, 1991.

[13] S.P. Meyn and R.L. Tweedie, "Computable bounds for convergence rates of Markov chains". Tech. Rep., Dept. of Statistics, Colorado State University, 1993.

[14] Jayant P. Pawar, Dr. Prashant V. Ingole, "Dynamic Spectrum Access Analysis in Cognitive Radio Based Wireless Regional Area Network", IEEE Xplore:, ISCO 2016, Vol 2, pp 479-482, , November 2016.

[15] Saroja T.V, Dr. Lata L. Ragha, Prof. Satyendra Kumar Sharma, "Hybrid spectrum access model using game theory approach for multi-channel heterogeneous mobile cognitive radio wireless sensor network", Indonesian Journal of Electrical Engineering and Computer Science (IJEECS), Vol. 16, No. 1, pp. 116-126, October 2019.

[16] Amira Osama, Heba A. Tag El-Dien, Ahmad A. Aziz El-Banna, Adly Tag El-Din "Spectrum sensing in single channel and multi-channel cognitive radio networks", Indonesian Journal of Electrical Engineering and Computer Science (IJEECS), Vol. 16, No. 2, pp. 812-817, November 2019.

[17] Dinh-ThuanDo, Chi-Bao Le, Anh-Tu Le, "Cooperative underlay cognitive radio assisted NOMA: secondary network improvement and outage performance"TELKOMNIKA (Telecommunication, Computing, Electronics and Control), Vol. 17, No. 5, pp. 2147-2154, October 2019.

[18] Wireless 2025: The Future of Mobile, Telephony Online, http://telephonyonline.com/, April 2009.

[19] J. R. Jackson, "Networks of waiting lines," Op. Res., vol. 5, pp. 518-521, 1957. 
[20] H. C. White and L. S. Christie, "Queuing with preemptive priorities or with breakdown," Op. Res., vol. 6, pp. $79-95$

[21] R. G. Miller, “Priority queues,” Ann. Math. Stat., vol. 31, no. 1, pp. 86-103, 1960.

[22] B. Avi-Itzhak and P. Naor, "On a problem of preemptive priority queuing," Op. Res., vol. 9, no. 3, pp. 664-672, 1961.

[23] A. Economou, "A characterization of product-form stationary distributions for queuing systems in random environment," International J. Simulation, vol. 4, pp. 24-31, 2003.

[24] I. Mitrani and B. Avi-Itzhak, "A many-server queue with service interruptions," Op. Res., vol. 16, no. 3, pp. 628-638.

[25] S. Wang, J. Zhang, and L. Tong, "Delay analysis for cognitive radio networks with random access: a fluid queue view, " in Proc. 2010 IEEE Infocom, pp. 1-9, 2010.

\section{BIOGRAPHIES OF AUTHORS}

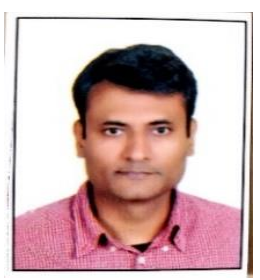

Jayant P. Pawar acquired his B.E. (EXTC) and M.E. (Digital Electronics) degrees from College of Engineering, Badanera, Amaravati (M.S.), Indiain 1994 and 2007, respectively. He is pursuing his Ph.D. from SGB Amaravati University, India. His areas of interest are wireless networks, Cognitive Radio and signal processing. He was development engineer at ESD, Pune for two years. He was H.O.D. at Atharva college of Engineering, Mumbai. He is the faculty at Rajeev Gandhi College of Engineering \& Technology, Pondicherry. He has 23 years of university level teaching experience in electronics and telecommunications .He had presented several papers in national and international conferences, and published papers in national and international journals.

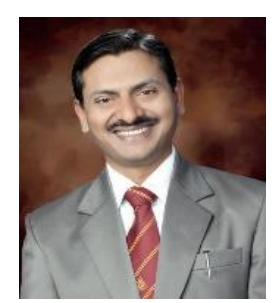

Prashant V. Ingole acquired his B.E. (EXTC) and M.E. (Automatic Control and Robotics) degrees from College of Engineering, Badanera, Amaravati (M.S.) and Faculty of Engineering \&Techenology, MS University, Baroda (Gujarat) in 1988 and 1993, respectively. He did his Ph.D. from Visvesvaraya National Institute of Technology, Nagpur, India, in 2010. His areas of interest are wireless networks, Digital signal processing, Digital Image Processing, Embedded systems and IoT. He was principle at G.H. Raisoni college of Engineering and Management, Amaravati from 2011 to 2017.He is currently working as a Professor and Head of the Information Technology Department, PRMIT\&R, Badnera- Amaravati, Maharastra. Presently he is the chairman of IETE Amaravati Centre.Email: pvingole@ mitra.ac.in 